\title{
On the Anomalous Change of Viscosity of Liquid Iron with Temperature*
}

\author{
By Yoshikiyo OGINO,,* Fritz O. BORGMANN, ${ }^{* * *}$ and Martin G. FROHBERG ${ }^{* * *}$
}

\section{Synopsis}

The viscosity of liquid iron containing 40 to 1230 ppm of oxygen was precisely measured. An anomalous change of the viscosity with temperature was observed at $1590^{\circ}$ to $1630^{\circ} \mathrm{C}$, depending on the oxygen concentration of the liquid. Below these temperatures, the viscosity deviates to higher values than that extrapolated from the higher temperatures assuming Arrhenius-type equation. With increase of oxygen, the viscosity decreased but the activation energies for viscous flow increased.

\section{Introduction}

Many investigations have been made on physical properties of liquid iron not only because of the technological importance but also of the scientific interest.

A few investigators have observed some characteristic behaviour on properties of liquid iron which suggest the abrupt structural change in liquid iron with temperature. Vertman and Samarin ${ }^{1)}$ observed on magnetic susceptibility an anomalous change at temperatures around $1670^{\circ} \mathrm{C}$ and interpreted this as due to the structural transition from $\delta$ to $\gamma$-iron like structure. Ferrier and Olette ${ }^{2)}$ observed a change of enthalpy function at temperatures near the melting point, which suggested that a ordered structure develops at the temperatures. Krieger and Trenkler ${ }^{3)}$ suggested from the compositional dependence of viscosity of $\mathrm{Fe}-\mathrm{C}$ and $\mathrm{Fe}-\mathrm{Ni}$ alloys that liquid iron have $\delta$-iron like structure. More recently, Vatolin and Pastuhov ${ }^{4}$ indicated from the direct $\mathrm{X}$-ray analysis that a structural transition from $\delta$ to $\gamma$-iron like structure takes place in liquid iron.

With regard to magnetic susceptibility, however, other investigators ${ }^{5-8)}$ did not observe the anomaly as observed by Vertman and Samarin. Urbain and Überlacher ${ }^{6,8)}$ suggested from their measurement that liquid iron should have the structure of fairly close packing of atoms having the coordination number of 10 to 12 . Thus, the previous works are contradicted themselves with respect to the structure and the related properties of liquid iron.

On the other hand, Ogino et al..$^{9,10)}$ have observed an anomalous change on viscosity and density of liquid iron at temperatures around $1600^{\circ} \mathrm{C}$. In spite of many measurements by other investigators, however, no one has observed such an anomaly. Then, in order to reconfirm the existence of the anomalous change and also to obtain more detailed informations, viscosity of liquid iron was precisely measured.

\section{Experimental Procedure}

The oscillating crucible method of the inverse suspending type was used for the measurement of viscosity. The details of the apparatus have been explained in the previous work ${ }^{11)}$ and will not be repeated here.

It is primarily important with this method to select the most suitable values of the oscillating period and the logarithmic decrement. To avoid the turbulent flow in the liquid, it seems desirable that the oscillating period is longer than about $6 \mathrm{sec}^{12}$ ) At present experiments, the values of 8.77 seconds and about 0.02 were chosen as the period and logarithmic decrement, respectively, by settling the moment of inertia of the oscillating system to about $3200 \mathrm{~g} \cdot \mathrm{cm}^{2}$.

The atmosphere is also an important factor, because it strongly affects the experimental accuracy especially at high temperatures. At the present experiment, the purified herium, in some cases mixed with about $10 \%$ hydrogen, was used under $1 \mathrm{~atm}$ pressure. It is known that the turbulent effect on the oscillation is much smaller in the case of herium than argon. ${ }^{12)}$

As the vessel for the liquid, alumina crucible of the size of about $3.5 \mathrm{~cm}$ dia and $6.0 \mathrm{~cm}$ depth were used. The volume of the liquid was $32.995 \mathrm{~cm}^{3}$, the depth in the crucible being about $3.3 \mathrm{~cm}$.

The measurements were made on several different concentrations of oxygen of about 40 to $1230 \mathrm{ppm}$. Electrolytic iron was melted in vacuum and deoxidized with small amounts of aluminium. In order to obtain the desired concentration of oxygen, the vacuummelted iron was further melted before the measurement in atmospheres of various oxygen partial pressures. After cutting off the oxide layer covering the surface, the iron was supplied to the measurement. Although the measurements were made under the controled atmospheres, the change of oxygen concentrations during the melting was unavoidable. Therefore, the melted iron was equilibrated at temperatures around $1600^{\circ} \mathrm{C}$ for a few huors before the measurement, and the oxygen analysis after the measurement was taken as that of the specimen.

To obtain the temperature dependence of viscosity very precisely, it was desired to make the measurement at very small temperature intervals. On account of this requirement, the measurements were made on continuously heating and cooling processes with the rate of $1^{\circ} \mathrm{C} / \mathrm{min}$. One measurement required about $3 \mathrm{~min}$. It has been preliminary confirmed that the viscosity values measured with such a manner are

* Received June 21, 1973.

** Formerly Institüt für Metallurgie-Allgemeine Metallurgie, Technische Universität Berlin, Berlin, Joachimstaler Strasse 31/32, West Germany, now at Department of Material Science, Himeji Instutute of Technology, Shosha, Himeji 671-22.

*** Institüt für Metallurgie-Allgemeine Metallurgie, Technische Universität Berlin, Berlin, Joachimstaler Strasse $31 / 32$, West Germany. 
quite same as that measured at a constant temperature. On cooling operations, liquid iron was super-cooled down to about $1450^{\circ} \mathrm{C}$, and the measurements were also made on these super-cooled iron melts.

\section{Calculation of Viscosity from Logarithmic Decrements}

In order to obtain viscosity coefficients from measured logarithmic decrements, the following equation proposed by Knappwost ${ }^{13)}$ has been used in most of the previous works.

$$
\lambda=\alpha \sqrt{\rho \eta}
$$

Where $\lambda$ is logarithmic decrement, $\eta$ and $\rho$ are viscosity and density of the liquid, respectively, and $\alpha$ is a constant. This equation, however, is a rather crude approximation and applicable only under limited experimental conditions. As has recently been criticized by Kleischmit and Grothe. ${ }^{14)}$ The necessary conditions would not necessarily be satisfied in experiments usually made. At the present experiments, therefore, the applicability of this equation was preliminary examined in accordance with the discussion by Kleischmit and Grothe, and it was concluded that Knappwost's equation can not be used for the measurement on liquid iron as explained below.

The following equations are those theoretically introduced by Roscoe $\mathrm{e}^{15,16)}$ and their validity has been confirmed in so called absolute measurement of viscosity;

$$
\begin{aligned}
\lambda / \rho & =\mathrm{A}(\eta / \rho)^{1 / 2}-\mathrm{B}(\eta / \rho)+\mathrm{C}(\eta / \rho)^{3 / 2} \\
\mathrm{~A} & =\frac{\pi^{3 / 2}}{I}\left(1+\frac{R}{4 H}\right) H R^{3} \tau^{1 / 2} \ldots \ldots \ldots \\
\mathrm{B} & =\frac{\pi}{I}\left(\frac{3}{2}+\frac{4}{\pi} \frac{R}{H}\right) H R^{2} \tau \ldots \ldots \ldots \ldots \\
\mathrm{C} & =\frac{\pi^{1 / 2}}{I}\left(\frac{3}{8}+\frac{9}{4} \frac{R}{H}\right) H R \tau^{3 / 2}, \ldots \ldots
\end{aligned}
$$

where $R$ and $H$ are inner radius of crucible and the depth of the liquid, respectively, and $I$ and $\tau$ are the moment of innertia and the oscillating period. By comparing these equations with Eq. (1) the following equation is obtained.

$$
\alpha=\mathrm{A}-\mathrm{B}(\eta / \rho)^{1 / 2}+\mathrm{C}(\eta / \rho) \cdots
$$

The Eq. (1) can be used only when the second and third terms are negligibly small compared with the first term. Then, the constants, A, B, and C, were calculated by using Eqs. (3) to (5), and the relation between $\alpha$ and $\eta / \rho$ was obtained as shown in Fig. 1. From this relation, it is clear that $\alpha$ can not be assumed as a constant at the present experimental conditions. The kinematic viscosity of liquid iron, as compared in Fig. 1, is considerably greater than that of other liquids such as mercury, tin, lead and copper, which are generally used to determine the constant $\alpha$. Accordingly, if Knappwost's equation was used, the error in the viscosity should amount to $20 \%$ or more. It must be noted that such a situation should come not only under the present experiments but also under almost all of the measurements previously made on iron, because, in order to make $\alpha$ constant, unrealistically large diameters of the crucible or too small oscillating periods must be employed.

At the present experiments, however, it is unrealistic to speak of absolute measurements because of the irregularity in the shape of the alumina crucibles. Therefore, the following semi-empirical procedure was employed.

As the standard liquid, mercury was chosen. From the logarithmic decrement, the oscillating period and the size of crucible, the value of $I$ was calculated using Eqs. (2) to (5). Then, from these unmerical values and Eqs. (3) to (5), the three constants, A, B, and C, were calculated. Equation (7) is the numerical equation there obtained.

$$
\frac{\lambda}{\rho}=0.0455\left(\frac{\eta}{\rho}\right)^{1 / 2}-0.0827\left(\frac{\eta}{\rho}\right)+0.0560\left(\frac{\eta}{\rho}\right)^{3 / 2}
$$

The above procedure means that a point in the function is determined experimentally with mercury and the functional form is approximately obtained from the theoretical equations.

To see the validity of such a procedure, Eq. (7) was applied to the measurement of other liquids, such as tin, copper, and distilled water. As the standard values of viscosity and density, values in Table 1 were used. Figure 2 shows the relation between $\lambda$ and $\sqrt{\rho \eta}$. In this figure, the slopes of straight-lines were calculated for each of the liquids from Eq. (6) with the numerical constants in Eq. (7). As may be seen, the experimental points do not conform to a straight-line but deviate just as estimated from Eq. (6). On the other hand, if the relation $\lambda / \rho$ vs. $\eta / \rho$ is plotted, as shown in Fig. 3, it well agrees with that calculated from Eq. (7). From these facts, it is clear that the use of Eq. (7) is much better in the accuracy than that of Knappwost's equation. In Table 1, the viscosities obtained by the above procedure are compared with the literature values.

The values of the three constants should change every crucibles. Therefore, the constants were determined for each of the used crucibles.

Before the calculation of viscosities from logarithmic decrements, the decrements must be corrected with the thermal expansions of the liquid and also of the crucible. The equations for these corrections are obtained from Eq. (2) to (5), taking only the first term

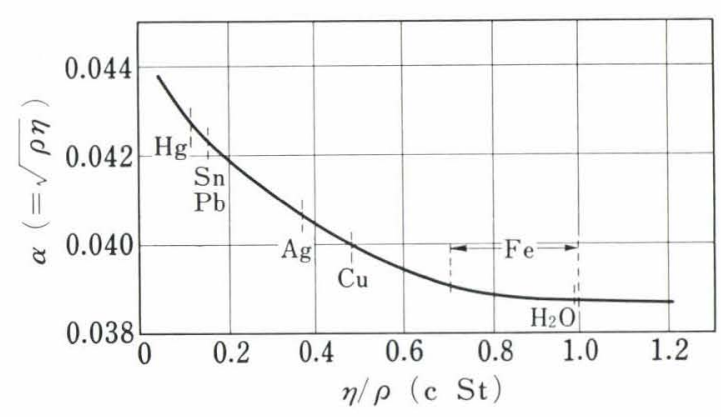

Fig. 1. Relation between $\alpha$ and $\eta / \rho$ 
in Eq. (2) because the other two terms can be neglected in these corrections.

The change of logarithmic decrements due to the thermal expansion of crucibles is approximately given by the formula,

$$
\frac{\Delta \lambda}{\lambda}=\frac{\left(1+\frac{R}{C}\right)}{\left(1+\frac{R}{4 C}\right)} \beta \Delta T,
$$

where $\mathrm{C}\left(=R^{2} H\right)$ is a constant, $\beta$ is the thermal ex-

Table 1. Comparison of viscosities obtained at present with literature values

\begin{tabular}{|c|c|c|c|c|}
\hline \multirow[b]{2}{*}{$\begin{array}{l}\text { Temperature } \\
\left({ }^{\circ} \mathbf{C}\right)\end{array}$} & \multirow[b]{2}{*}{$\begin{array}{l}\text { Density } \\
\left(\mathrm{g} / \mathrm{cm}^{3}\right)\end{array}$} & \multicolumn{2}{|c|}{ Viscosity $(c \mathrm{P})$} & \multirow{2}{*}{$\begin{array}{c}\text { Loga- } \\
\text { rithmic } \\
\text { decrement }\end{array}$} \\
\hline & & $\begin{array}{l}\text { Literature } \\
\text { value }\end{array}$ & $\begin{array}{l}\text { Present } \\
\text { experi- } \\
\text { ment }\end{array}$ & \\
\hline Tin & Ref. 17) & Ref. 18) & & \\
\hline 500 & 6.780 & 1.133 & 1.135 & 0.0117 \\
\hline 600 & 6.704 & 1.021 & 1.006 & 0.0110 \\
\hline 700 & 6.631 & 0.941 & 0.903 & 0.0104 \\
\hline 800 & 6.559 & 0.880 & 0.823 & 0.0099 \\
\hline Copper & Ref. 19) & Ref. 11) & & \\
\hline 1130 & 8.00 & 3.68 & 3.577 & 0.0215 \\
\hline 1140 & 7.99 & 3.61 & 3.545 & 0.0214 \\
\hline 1160 & 7.97 & 3.47 & 3.443 & 0.0211 \\
\hline 1180 & 7.96 & 3.33 & 3.338 & 0.0208 \\
\hline Mercury & Ref. 20) & Ref. 20) & & \\
\hline 15 & 13.53 & 1.525 & 1.525 & 0.01942 \\
\hline Water & Ref. 20) & Ref. 20) & & \\
\hline 15 & 0.997 & 0.865 & 0.833 & 0.00350 \\
\hline
\end{tabular}

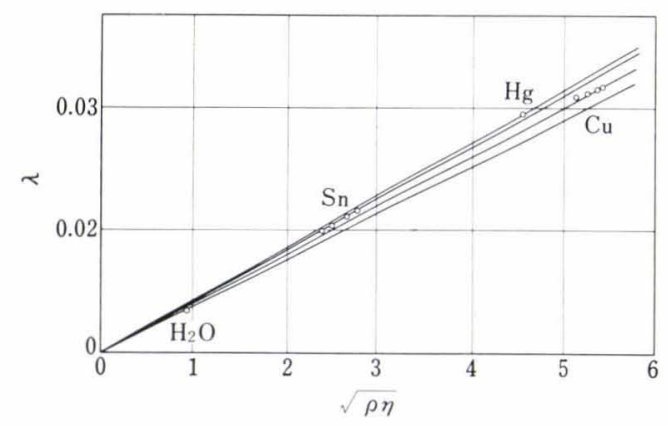

Fig. 2. Relation between $\lambda$ and $\sqrt{\rho \eta}$, the lines being calculated from Eq. (7)

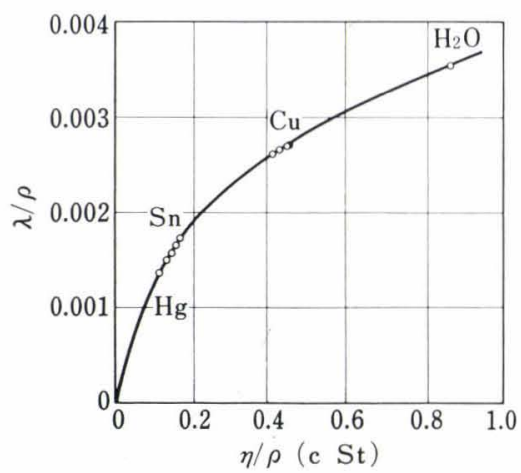

Fig. 3. Relation between $\lambda / \rho$ and $\eta / \rho$, the curve being calculated from Eq. (7) pansion coefficient of alumina. This correction amounts to about $3.4 \%$ in the change of viscosity in the case of liquid iron.

After this correction, the correction for the thermal expansion of the liquid was made. This crrection can be made by the formula,

$$
\frac{\Delta \lambda}{\lambda}=\frac{\Delta H}{H+\frac{R}{4}}
$$

where $\Delta H$ is the change in the depth of the liquid and can be calculated from the density change of the liquid.

As the density of liquid iron, which is necessary for the later correction and also for the calculation of viscosity from logarithmic decrements, the values by Lucas $^{22)}$ were used as most reliable values obtained up to date.

\section{Experimental Results}

The temperature dependence of viscosity of liquid iron is shown in Fig. 4 for the different concentrations of oxygen from 40 to $1230 \mathrm{ppm}$. Figure 5 also shows the temperature dependence in the relation between $\log$ viscosity and the reciplocal of the absolute temperature. In Fig. 5, it seems that a straight-line relationships does not prevail over the entire range of temperatures examined. However, at temperatures higher than that slightly above the temperatures indicated by arrows, the straight-line relationships are apparently satisfied. Therefore, the equations to express the straight-lines were obtained in the high temperature range by the least square method. The standard deviation of the measured values from the least square values was calculated to $0.019 \mathrm{cP}$ in the high temperature range. As may be seen in Fig. 5, the viscosity begins to deviate to higher values from the temperatures indicated by arrows. It must be noticed, furthermore, that a saddle like change can be seen on each of the curves at the temperatures indicated by arrows in Figs. 4 and 5. Although this change only slightly appears, its existence seems to be

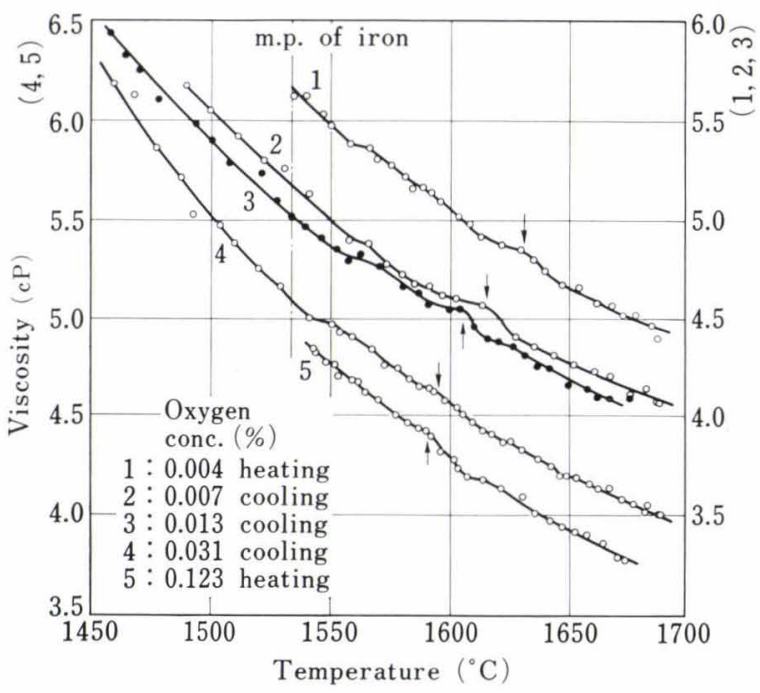

Fig. 4. Temperature dependency of viscosity of liquid iron 
undoubted judging from the comparison with the value of the standard deviation. Also at temperatures below the saddle like change, some other complexed features might be seen on the curves. The temperature of the saddle like change, from which the deviation from the straight-line relationship begins, decreases with increase of the oxygen concentrations, as shown in Fig. 6. At 40 ppm of oxygen, the change occurs at about $1630^{\circ} \mathrm{C}$. With increase of oxygen to 200 to $300 \mathrm{ppm}$, the temperature rapidly decreases to about $1595^{\circ} \mathrm{C}$, and then gradually decreases at the higher concentrations.

Thus, conflicting with previous works by other investigators on the viscosity of liquid iron, the present results have shown an anomalous behaviour of the viscosity, i.e., the deviation from the Arrhenius-type equation accompanied by a saddle like change.

On the other hand, the effect of the dissolved oxygen on the viscosity of liquid iron is also interesting. As

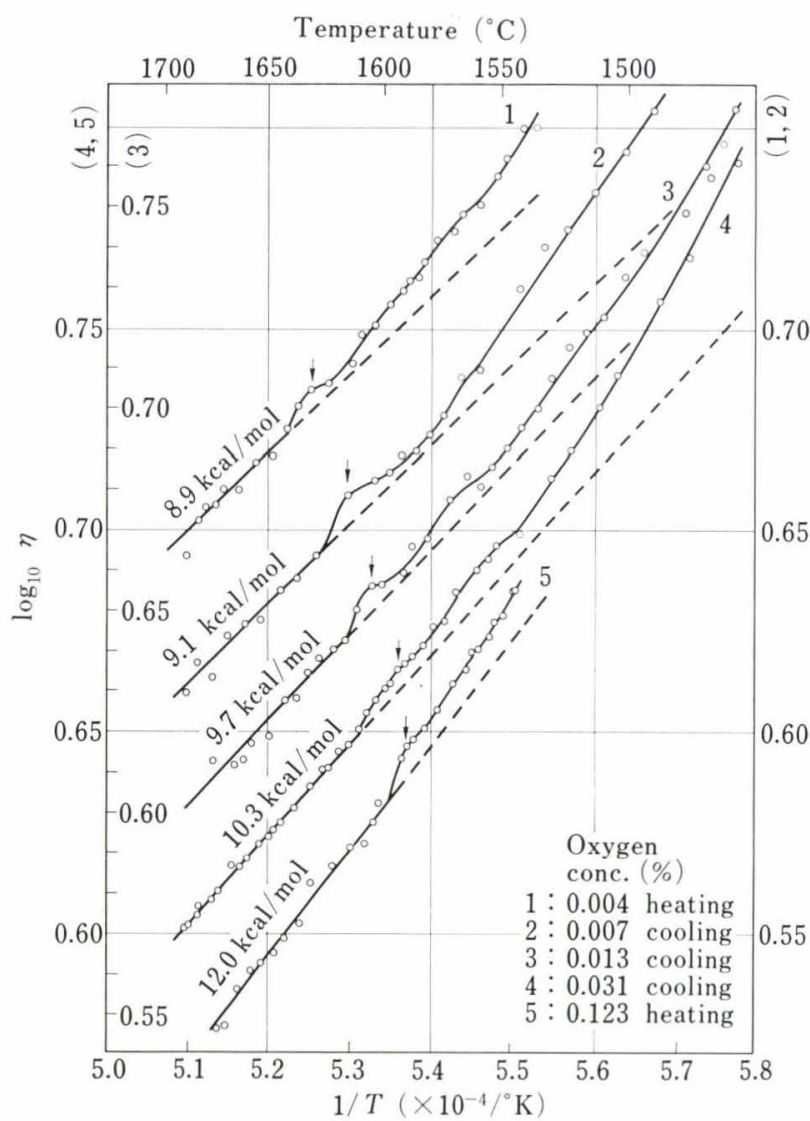

Fig. 5. Relation between $\log \eta$ and $1 / T$

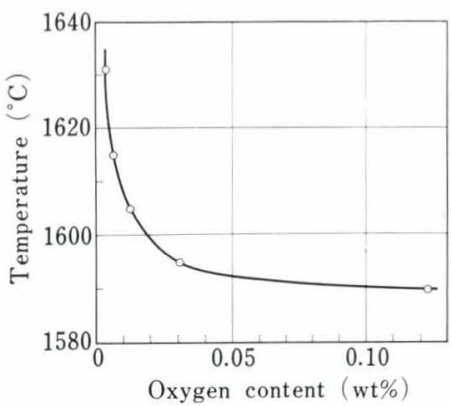

Fig. 6. Effect of oxygen in liquid iron on temperatures of anomalous change shown in Fig. 7 together with the present result, the previous works have contradicted themselves with respect to the dependency of the viscosity on the oxygen concentration. At the present experiment, the viscosity decreased with increasing oxygen concentration to about $100 \mathrm{ppm}$ and then gradually up to 1230 ppm. Such a trend is quite similar with that observed by Romanov and Kochegarov. ${ }^{23)}$ On the contrary, the apparent activation energy at the high temperature range increased with increase of oxygen, as shown in Fig. 8.

\section{Discussion of Results}

In previous works on the viscosity of liquid iron, it has usually been assumed that the temperature dependence of the viscosity follows an Arrhenius-type equation in the whole temperature range examined. The present observation conflicts to the previous one with this respect. The present observation, however, quite agrees with the result of experiment previously made by one of the authors ${ }^{9)}$ under an another experimental conditions; i.e., the similar anomalous change was observed at about $1600^{\circ} \mathrm{C}$ on liquid iron containing about 200 ppm of oxygen. In the previous experiment, the viscosity of liquid nickel was also measured at nearly the same temperatures, but no anomaly was observed in this case.

Some erroneous causes, such as reactions between the alumina crucible and the liquid, the change of oxygen concentrations, and of the meniscus of the liquid during the measurement might be considered

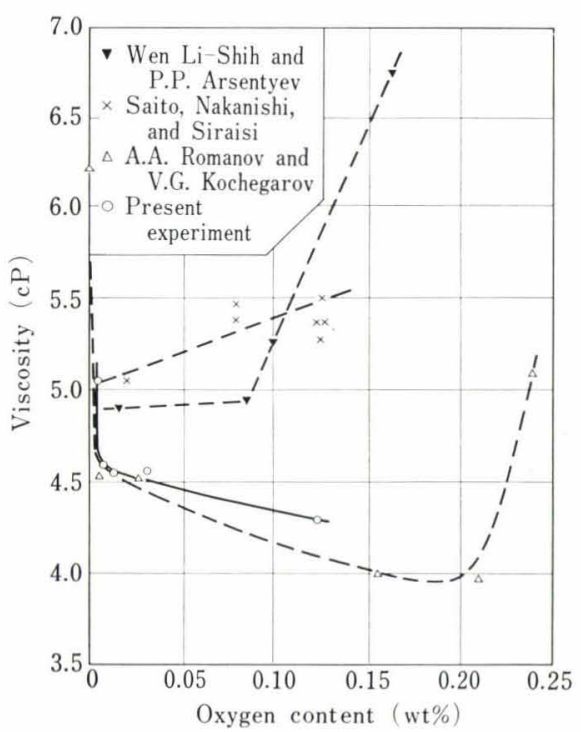

Fig. 7. Effect of oxygen on viscosity of liquid iron at $1600^{\circ} \mathrm{C}$

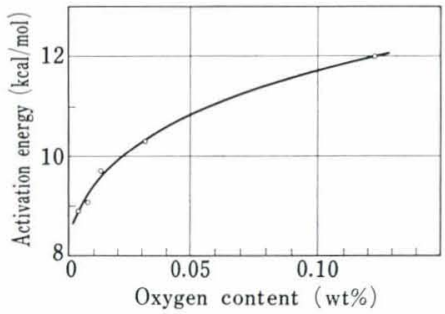

Fig. 8. Relation between activation energy of viscous flow and oxygen concentration of liquid iron 
to affect more or less the experimental results. However, it is very improbable that the observed anomaly has arised from the effect of them, because the anomaly appears quite reversibly on the heating and cooling operations. The effect of impurity elements is a difficult problem to estimate. Oxygen will be the most important of them because of its high reactivity with other elements, and in fact the anomaly is strongly dependent on the oxygen concentration. The fact, however, that oxygen lowers the temperature of the anomalous change seems incompatible with the assumption that oxygen is the essential factor for the occurence of the anomaly. It must be stressed, furthermore, the similar anomalies have been observed also in other properties such as the density ${ }^{10}$ ) and magnetic susceptibility. ${ }^{11}$ Thus, it seems probable that the anomalous change observed at present arised from the structural transition of liquid iron.

As well known, on X-ray analysis, the structural factor, $a(K)$, of liquid metals commonly shows a close resemblance with that of hard sphere liquid. However, when there exists the directional nature in the interatomic bond, an ordering trend may be expected in the liquid. In the case of $\mathrm{Sb}, \mathrm{Bi}$, and $\mathrm{Sn}$, for example, an additional small peak appears on $a(K)$ indicating the existence of the local order characteristic to the elements. It would not be unreasonable to expect such an ordering trend also in the case of liquid iron, because in this metal, the directional $d$ electron bonding may contribute to the total bond, resulting in b.c.c. structure in the solid state.

Now in Fig. 5, it is clear that an Arrhenius-type equation is not applicable in the range of lower temperatures than that indicated by arrows. However, if the straight-line relationships are approximately assumed at temperatures lower than about $1550^{\circ} \mathrm{C}$, apparent activation energies are obtained to 13.7, 14.7, and $17.1 \mathrm{kcal} / \mathrm{mol}$ in the cases of 70,130 , and $310 \mathrm{ppm}$ of oxygen, respectively. These values are 1.5 times larger than that at the range of higher temperatures. According to the theory by Frenkel ${ }^{25)}$ and Eyring, ${ }^{26)}$ the activation energy for viscous flow is equivalent to the energy necessary to form the hole in the liquid. It should not be expected, however, that the structural change in the liquid causes such a large difference in the activation energy. Therefore, as the reason for the increment of viscosity at the lower temperatures, it may be assumed that some particles of sizes larger than iron atom are suspended in the liquid and increase the viscosity by a mechanism similar to that of Einstein's suspension viscosity. ${ }^{27}$ ) As these particles, clusters of iron atoms may be assumed. If these clusters can be regarded as rigid spheres, their amounts can be evaluated by applying Einstein's equation. Then, as an attempt, a calculation was made assuming that the increase of viscosity from the values extrapolated from the high temperature region is mainly due to the cluster like rigid spheres. The following equation. ${ }^{28)}$ was used,

$$
\frac{\eta}{r_{0}}=1+2.5 \phi+7 \phi^{2}
$$

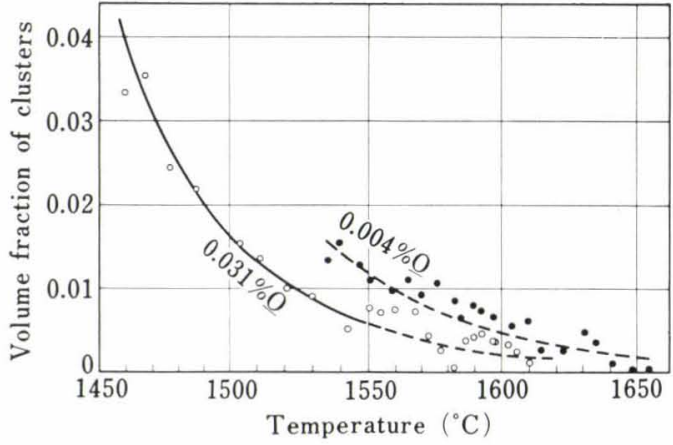

Fig. 9. Temperature dependency of volumetric fraction of clusters calculated from Eq. (10)

where $\eta_{0}$ and $\eta$ are the actual and the extrapolated values of viscosity. $\phi$ is the volumetric fraction of clusters. As shown in Fig. 9, the volumetric fraction of clusters thus obtained are relatively small, i.e., 0.016 and 0.008 at the melting point in the case of 40 and $310 \mathrm{ppm}$ of oxygen, respectively. Furthermore, assuming that the heat of formation of the clusters is equal to the heat of fusion of $\delta$-iron, $3.6 \mathrm{kcal} / \mathrm{mol}$, the mean number of atoms contained in a cluster was calculated by a procedure similar to that used by Mclaughlin and Ubbelohde. ${ }^{28}$ ) The value obtained is about 40 atoms in both cases of 40 and $310 \mathrm{ppm}$ of oxygen. These values are seemingly not unreasonable as the amounts and the size of the clusters.

\section{Summary}

The viscosity of liquid iron containing 40 to 1230 ppm of oxygen was measured by the use of oscillating crucible method. In a preliminary experiment, it was clarified that the use of Knappwost's equation lead to a significant error in the measurement on liquid iron. At the present experiment, therefore, an improved equation with three constants based on Roscoe's equation was applied. The main results may be summarized as follows.

(1) An anomalous change in the viscosity with temperature was observed on liquid iron at temperatures around $1600^{\circ} \mathrm{C}$. Below these temperatures, the viscosity deviates to higher values than that extrapolated from the higher temperatures assuming Arrhenius-type temperature dependence. The temperature of the anomalous change depends on the oxygen concentrations of liquid iron; i.e., about $1630^{\circ} \mathrm{C}$ for $40 \mathrm{ppm}$ of oxygen and rapidly decreases to about $1600^{\circ} \mathrm{C}$ with increase of oxygen to about $200 \mathrm{ppm}$. Such an anomalous change would suggest that certain structural transition took place in liquid iron.

(2) The viscosity of liquid iron decreased rapidly with increase of oxygen concentration from about 40 to $200 \mathrm{ppm}$ and then gradually decreased up to about $1230 \mathrm{ppm}$. On the contrary, the activation energies for viscous flow increased with increase of the oxygen concentrations.

\section{Acknowledgements}

The authors are thankful to Deutsche Forschungsgemeinschaft (BRD) for the financial support of this work. One of the authors, Y.O., is also thankful to 
Alexander von Humboldt Stiftung for providing financial support during his stay in Germany.

\section{REFERENCES}

1) A. A. Vertman and A. M. Samarin: Dokl. Akad. Nauk SSSR, 134 (1960), 326; A. M. Samarin: J.I.S.I., 200 (1962), 95.

2) A. Ferrier and M. Olette: Compt. Rend., 254 (1962), 2322.

3) W. Krieger and H. Trenkler: Arch. Eisenhüttenw., 3 (1971), 175.

4) N. A. Vatolin and E. A. Pastuhov: The Fourth Joint Symposium of USSR and Japan on Physical Chemistry of Metallurgical Processes, (1973), held in Tokyo.

5) Y. Nakagawa: J. Phys. Soc. Japan, 11 (1956), 855.

6) E. Wachtel and G. Urbain: Compt. Rend., 255 (1962), 2382.

7) R. Kohlhaas and H. Lange: Z. Ang. Phys., 516 (1965), 453.

8) G. Urbain and E. Überlacher: Adv. Phys., 16 (1967), 429.

9) Y. Ogino, Z. Morita, T. Maehana, K. Yokotani, and A. Adachi: Tetsu-to-Hagané, 56 (1970), 1633; Y. Ogino, Z. Morita and A. Adachi: Tech. Rept. Osaka Univ., 21 (1971), 399.

10) Z. Morita, Y. Ogino, H. Kaito, and A. Adachi: J. Japan Inst. Metals, 34 (1970), 248.

11) H. Schenck, M. G. Frohberg, and K. Hoffmann: Arch. Eisenhüttenw., 2 (1963), 93.

12) Z. Morita, Y. Ogino, T. Iba, T. Maehana, and A. Adachi:
Tetsu-to-Hagané, 56 (1970), 1613.

13) A. Knappwost: Z. Metallk., 39 (1948), 314; Z. Phys. Chem., 200 (1952), 81.

14) P. Kleischmit and K. H. Grothe: Z. Metallk., 61 (1970), 976.

15) R. Roscoe: Proc. Phys. Soc., 72 (1958), 576.

16) R. Roscoe and W. Bainbridge: Proc. Phys. Soc., 72 (1959), 585.

17) L. D. Lucas: Mem. Sci. Rev. Met., 61 (1964), 1.

18) M. Menz and F. Sauerwald: Acta Met., 14 (1966), 1617

19) G. Urbain and L. D. Lucas: Proc. Nat. Phys. Lab. Symp., No. 9, (1959), London.

20) Kagaku-Binran, (1958), Nippon Kagakukai.

21) L. D. Lucas: Compt. Rend., 250 (1960), 1850.

22) Wen Li-Shih and P. P. Arsentyev: Izv. VUZov, Cher. Met., 7 (1961), 5.

23) A. A. Romanov and V. G. Kochegarov: Izv. Akad. Nauk SSSR Met. i Gorn Delo., 3 (1964), 63.

24) T. Saito, K. Nakanishi, and Y. Shiraishi: J. Japan Inst. Metals, 31 (1967), 881.

25) J. Frenkel: Kinetic Theory of Liquid, (1955), Dover Publications.

26) S. Glasstone, K.J. Laidler, and H. Eyring: The Theory of Rate Processes, (1941), McGraw-Hill Book Company, New York

27) A. Einstein: Ann. Physik, 19 (1906), 289; 34 (1911), 591.

28) E. Mclaughlin and A. R. Ubbelohde: Trans. Faraday Soc., 54 (1958), 1804. 